\title{
PARTICLE IN A UNIFORM FIELD IN A NONCOMMUTATIVE SPACE WITH PRESERVED TIME REVERSAL AND ROTATIONAL SYMMETRIES
}

\author{
Kh. P. Gnatenko(D), Kh. I. Stakhur, A. V. Kryzhova \\ Ivan Franko National University of Lviv, \\ Department for Theoretical Physics, \\ 12, Drahomanov St., Lviv, UA-79005, Ukraine \\ khrystyna.gnatenko@gmail.com
}

(Received 18 January 2021; in final form 23 March 2021; accepted 24 March 2021; published online 31 May 2021)

\begin{abstract}
The paper studies quantized space described by a time reversal invariant and rotationally invariant noncommutative algebra of a canonical type. A particle in a uniform field is considered. We find exactly the energy of a particle in a uniform field in the quantized space and its wavefunctions. It is shown that the motion of the particle in the field direction in time reversal invariant and rotationally invariant noncommutative space is the same as in an ordinary space (a space with the ordinary commutation relations for the operators of coordinates and the operators of momenta). The noncommutativity of the coordinates has an influence only on the motion of the particle in the directions perpendicular to the field direction. Namely, space quantization has an effect on the mass of the particle.
\end{abstract}

Key words: quantized space, noncommutative coordinates, time reversal symmetry, rotational symmetry, particle in uniform field.

DOI: https://doi.org/10.30970/jps.25.2002

\section{INTRODUCTION}

According to the String theory and Quantum gravity, a minimal length of the order of the Planck length exists $[1,2]$. A space with the minimal length can be described on the basis of the idea of the deformation of the commutation relations for operators of coordinates and operators of momenta.

Many different deformed algebras leading to the minimal length were proposed. One can distinguish three types of the algebras: noncommutative algebras of the canonical type (see, for instance, [3-6]), noncommutative algebras of the Lie type (see, for instance, [7-9]), nonlinear deformed algebras (see, for instance, [10-12]). Noncommutative algebras of a canonical type are the most simple algebras which describe space quantization on the Planck scale. An algebra with the noncommutativity of coordinates of canonical type is characterized by the following relations

$$
\begin{aligned}
& {\left[X_{i}, X_{j}\right]=i \hbar \theta_{i j}} \\
& {\left[X_{i}, P_{j}\right]=i \hbar \delta_{i j},} \\
& {\left[P_{i}, P_{j}\right]=0}
\end{aligned}
$$

Here $\theta_{i j}$ are called parameters of noncommutativity which are elements of constant matrixes. Contrary to dimensionless $\delta_{i j}$ in (2), parameters $\theta_{i j}$ are dimensionful, $\hbar \theta_{i j}$ in (1) has the dimension of squared length. Properties of physical systems within the frame of noncommutative algebra of a canonical type have been widely studied (see, for instance, [3-6, 13-19], and references therein). Among the problems, a noncommutative gravitational quantum well has been examined [14, 18, 19]. It is worth stressing that in a noncommutative space characterized by commutation relations (1)-(3), the rotational and time reversal symmetries are not preserved [5, 20-22].
In [23] a noncommutative algebra which is rotationally invariant and equivalent to a noncommutative algebra of a canonical type was proposed. In [24] the effect of noncommutativity on the mass of a particle in a uniform field was found within the frame of the rotationally invariant noncommutative algebra of a canonical type [23].

In the present paper, we study a particle in a uniform field within the frame of the rotationally invariant and time reversal invariant noncommutative algebra of a canonical type proposed in [22]. The total Hamiltonian is constructed and analyzed. We find exactly the energy and wave functions of a particle in a uniform field in a noncommutative space with preserved rotational and time reversal symmetries.

The paper is organized as follows. In Section 2, a time reversal and rotationally invariant algebra with the noncommutativity of coordinates is presented. Section 3 is devoted to the studies of a particle in uniform field in rotationally invariant and time reversal invariant noncommutative space. Conclusions are presented in Section 4.

\section{TIME REVERSAL AND ROTATIONALLY INVARIANT ALGEBRA WITH THE CANONICAL NONCOMMUTATIVITY OF COORDINATES}

In [22] for preserving rotational and time reversal symmetries in a noncommutative space, the authors considered the idea to generalize parameters of noncommutativity, defining the tensor of the coordinate noncommutativity as

$$
\theta_{i j}=\frac{c_{\theta}}{\hbar} \sum_{k} \varepsilon_{i j k} p_{k}^{a}
$$


here $p_{i}^{a}$ are additional momenta governed by a rotationally invariant system, $c_{\theta}$ is a constant. For simplicity, in [22] the additional momenta were assumed to be governed by the harmonic oscillator

$$
H_{\mathrm{osc}}^{a}=\frac{\left(\mathbf{p}^{a}\right)^{2}}{2 m_{\mathrm{osc}}}+\frac{m_{\mathrm{osc}} \omega_{\mathrm{osc}}^{2} \mathbf{a}^{2}}{2} .
$$

Here $a_{i}$ are additional coordinates conjugated to momenta $p_{i}^{a}$. The following relations are satisfied

$$
\begin{aligned}
& {\left[a_{i}, p_{j}^{a}\right]=i \hbar \delta_{i j},} \\
& {\left[a_{i}, a_{j}\right]=\left[p_{i}^{a}, p_{j}^{a}\right]=0 .}
\end{aligned}
$$

The oscillator length is considered to be equal to the Planck length $\sqrt{\hbar} / \sqrt{m_{\mathrm{osc}} \omega_{\mathrm{osc}}}=l_{\mathrm{P}}$ and the frequency of the oscillator is assumed to be very large [22]. So, the harmonic oscillator (5) remains in the ground state.

The time reversal and rotationally invariant algebra with the canonical noncommutativity of coordinates reads

$$
\begin{aligned}
& {\left[X_{i}, X_{j}\right]=i c_{\theta} \sum_{k} \varepsilon_{i j k} p_{k}^{a},} \\
& {\left[X_{i}, P_{j}\right]=i \hbar \delta_{i j}, \quad\left[P_{i}, P_{j}\right]=0,} \\
& {\left[p_{i}^{a}, X_{j}\right]=\left[p_{i}^{a}, P_{j}\right]=0 .}
\end{aligned}
$$

It is convenient to represent coordinates and momenta which satisfy $(8),(9)$ by coordinates and momenta $x_{i}, p_{i}$ satisfying the ordinary commutation relations

$$
\begin{aligned}
& {\left[x_{i}, x_{j}\right]=\left[p_{i}, p_{j}\right]=0,} \\
& {\left[x_{i}, p_{j}\right]=i \hbar \delta_{i j} .}
\end{aligned}
$$

The representation is

$$
X_{i}=x_{i}+\frac{1}{2}[\boldsymbol{\theta} \times \mathbf{p}]_{i}, \quad P_{i}=p_{i},
$$

here $\boldsymbol{\theta}=\left(\theta_{1}, \theta_{2}, \theta_{3}\right)$,

$$
\theta_{i}=\frac{c_{\theta} p_{i}^{a}}{\hbar}
$$

From relation (10) it follows that algebra (8), (9) is equivalent to the algebra with the canonical noncommutativity of coordinates. Relations (8), (9) are invariant under the time reversal transformation which includes a complex conjugation. Also, after this transformation, the coordinates and the momenta change as $X_{i} \rightarrow X_{i}, P_{i} \rightarrow-P_{i}, p_{i}^{a} \rightarrow-p_{i}^{a}$. Algebra (8), (9) is time reversal invariant [22]. After rotation, the coordinates and momenta change as $X_{i}^{\prime}=U(\varphi) X_{i} U^{+}(\varphi)$, $P_{i}^{\prime}=U(\varphi) P_{i} U^{+}(\varphi) p_{i}^{a \prime}=U(\varphi) p_{i}^{a} U^{+}(\varphi), U(\varphi)=$ $\exp \left(i \varphi\left(\mathbf{n} \cdot \mathbf{L}^{\mathbf{t}}\right) / \hbar\right)$ with $\mathbf{L}^{\mathbf{t}}=[\mathbf{x} \times \mathbf{p}]+\left[\mathbf{a} \times \mathbf{p}^{a}\right]$. Commutation relations (8), (9) are invariant under rotation, the algebra is rotationally invariant [22].

In the next section, we study a particle in a uniform field within the frame of a rotationally invariant and time reversal invariant noncommutative algebra (8), (9).

\section{ENERGY OF A PARTICLE IN A UNIFORM FIELD IN A ROTATIONALLY INVARIANT AND TIME REVERSAL INVARIANT NONCOMMUTATIVE SPACE}

Let us study a particle with mass $m$ in uniform field with the following Hamiltonian

$$
H_{p}=\frac{P^{2}}{2 m}-\alpha X_{3} .
$$

In (15) the coordinates and momenta satisfy relations (8), (9). Without loss of generality, for convenience we consider the field pointed in the $X_{3}$ direction (in (15) $\alpha$ characterizes the force acting on the particle). For instance, for a particle in a uniform gravitational field $g$ with the direction along the $X_{3}$ axis, we have $\alpha=-m g$. In the case of a charged particle in a uniform electric field $E$ with the direction along the $X_{3}$ axis, the parameter $\alpha$ reads $\alpha=-q E$, where $q$ is the charge of the particle. Because algebra (8), (9) is rotationally invariant, the results of this section can be generalized to the case of an arbitrary direction of the field.

To construct a time reversal invariant and rotationally invariant noncommutative algebra (8), (9) additional momenta $p_{i}^{a}$ were involved, therefore, to study a particle in a uniform field in the space (8), (9) one should write the following Hamiltonian

$$
H=\frac{P^{2}}{2 m}-\alpha X_{3}+\frac{\left(p^{a}\right)^{2}}{2 m_{\mathrm{osc}}}+\frac{m_{\mathrm{osc}} \omega_{\mathrm{osc}}^{2} a^{2}}{2},
$$

the last two terms where correspond to harmonic oscillator (5). Then, to find the influence of the space quantization on the energy of a particle in a uniform field, it is convenient to use representation (13) and rewrite Hamiltonian (16) as follows

$$
\begin{aligned}
H & =\frac{p^{2}}{2 m}-\alpha x_{3}-\frac{\alpha}{2}[\boldsymbol{\theta} \times \mathbf{p}]_{3}+\frac{\left(p^{a}\right)^{2}}{2 m_{\mathrm{osc}}}+\frac{m_{\mathrm{osc}} \omega_{\mathrm{osc}}^{2} a^{2}}{2} \\
& =\frac{p^{2}}{2 m}-\alpha x_{3}-\frac{\alpha c_{\theta}}{2 \hbar}\left(p_{1}^{a} p_{2}-p_{2}^{a} p_{1}\right)+\frac{\left(p^{a}\right)^{2}}{2 m_{\mathrm{osc}}}+\frac{m_{\mathrm{osc}} \omega_{\mathrm{osc}}^{2} a^{2}}{2}
\end{aligned}
$$

Here we take into account (14). 
To find the exact expression for the energy of a particle in a uniform field in a space described by commutation relations (8), (9), we rewrite Hamiltonian (17) as

$$
\begin{aligned}
H & =\left(1-\frac{\alpha^{2} c_{\theta}^{2} m m_{\mathrm{osc}}}{4 \hbar^{2}}\right) \frac{p_{1}^{2}}{2 m}+\left(1-\frac{\alpha^{2} c_{\theta}^{2} m m_{\mathrm{osc}}}{4 \hbar^{2}}\right) \frac{p_{2}^{2}}{2 m}+\frac{p_{3}^{2}}{2 m}-\alpha x_{3} \\
& +\frac{1}{2 m_{\mathrm{osc}}}\left(p_{1}^{a}-\frac{\alpha c_{\theta} m_{\mathrm{osc}}}{2 \hbar} p_{2}\right)^{2}+\frac{1}{2 m_{\mathrm{osc}}}\left(p_{2}^{a}+\frac{\alpha c_{\theta} m_{\mathrm{osc}}}{2 \hbar} p_{1}\right)^{2} \\
& +\frac{\left(p_{3}^{a}\right)^{2}}{2 m_{\mathrm{osc}}}+\frac{m_{\mathrm{osc}} \omega_{\mathrm{osc}}^{2} a_{1}^{2}}{2}+\frac{m_{\mathrm{osc}} \omega_{\mathrm{osc}}^{2} a_{2}^{2}}{2}+\frac{m_{\mathrm{osc}} \omega_{\mathrm{osc}}^{2} a_{3}^{2}}{2}
\end{aligned}
$$

Note that operators

$$
\tilde{H}_{p}=\left(1-\frac{\alpha^{2} c_{\theta}^{2} m}{4 \hbar \omega_{\mathrm{osc}} l_{\mathrm{P}}^{2}}\right) \frac{p_{1}^{2}}{2 m}+\left(1-\frac{\alpha^{2} c_{\theta}^{2} m}{4 \hbar \omega_{\mathrm{osc}} l_{\mathrm{P}}^{2}}\right) \frac{p_{2}^{2}}{2 m}+\frac{p_{3}^{2}}{2 m}-\alpha x_{3},
$$

and

$$
\begin{aligned}
\tilde{H}_{\mathrm{osc}} & =\frac{1}{2 m_{\mathrm{osc}}}\left(p_{1}^{a}-\frac{\alpha c_{\theta}}{2 \omega_{\mathrm{osc}} l_{\mathrm{P}}^{2}} p_{2}\right)^{2}+\frac{1}{2 m_{\mathrm{osc}}}\left(p_{2}^{a}+\frac{\alpha c_{\theta}}{2 \omega_{\mathrm{osc}} l_{\mathrm{P}}^{2}} p_{1}\right)^{2} \\
& +\frac{\left(p_{3}^{a}\right)^{2}}{2 m_{\mathrm{osc}}}+\frac{m_{\mathrm{osc}} \omega_{\mathrm{osc}}^{2} a_{1}^{2}}{2}+\frac{m_{\mathrm{osc}} \omega_{\mathrm{osc}}^{2} a_{2}^{2}}{2}+\frac{m_{\mathrm{osc}} \omega_{\mathrm{osc}}^{2} a_{3}^{2}}{2}
\end{aligned}
$$

in (18) commute

$$
\left[\tilde{H}_{p}, \tilde{H}_{\mathrm{osc}}\right]=0
$$

Writing (19), (20) we take into account that

$$
\sqrt{\frac{\hbar}{m_{\mathrm{osc}} \omega_{\mathrm{osc}}}}=l_{\mathrm{P}}
$$

as was assumed in the paper [22], where the noncommutative algebra invariant upon time reversal and rotationally invariant was constructed.

Operator $\tilde{H}_{p}$ can be rewritten as

$$
\tilde{H}_{p}=\tilde{H}_{1}+\tilde{H}_{2}+\tilde{H}_{3}
$$

with

$$
\begin{aligned}
& \tilde{H}_{1}=\frac{p_{1}^{2}}{2 m_{\mathrm{eff}}}, \\
& \tilde{H}_{2}=\frac{p_{2}^{2}}{2 m_{\mathrm{eff}}}, \\
& \tilde{H}_{3}=\frac{p_{3}^{2}}{2 m}-\alpha x_{3}, \\
& {\left[\tilde{H}_{1}, \tilde{H}_{2}\right]=\left[\tilde{H}_{2}, \tilde{H}_{3}\right]=\left[\tilde{H}_{1}, \tilde{H}_{3}\right]=0 .}
\end{aligned}
$$

The effective mass reads

$$
\begin{aligned}
m_{\mathrm{eff}} & =m\left(1-\frac{\alpha^{2} c_{\theta}^{2} m m_{\mathrm{osc}}}{4 \hbar^{2}}\right)^{-1} \\
& =m\left(1-\frac{\alpha^{2} c_{\theta}^{2} m}{4 \hbar \omega_{\mathrm{osc}} l_{\mathrm{P}}^{2}}\right)^{-1} .
\end{aligned}
$$

Here we would like to mention that according to (28), the effective mass $m_{\text {eff }}$ is positive if $\alpha^{2} c_{\theta}^{2} m / 4 \hbar \omega_{\text {osc }} l_{\mathrm{P}}^{2}<$ 1. Let us analyze this inequality. Assuming that the minimal length for an electron is the Planck length, we have $c_{\theta}=2 l_{\mathrm{P}}^{3} / 3 \hbar$. To write this expression, we take into account that the minimal length is as follows $\sqrt[4]{3 \hbar^{2}\left\langle\theta^{2}\right\rangle} / \sqrt[4]{2}$, where $\left\langle\theta^{2}\right\rangle=\sum_{i}\left\langle\psi_{0,0,0}^{a}\left|\theta_{i}^{2}\right| \psi_{0,0,0}^{a}\right\rangle=$ $3 c_{\theta}^{2} / 2 l_{\mathrm{P}}^{2}, \psi_{0,0,0}^{a}$ is eigenfunction of harmonic oscillator (5) in the ground state (see [26]). Note that $\omega_{\text {osc }}$ is assumed to be very large [22]. Considering $\omega_{\mathrm{osc}}=1 / t_{\mathrm{P}}\left(t_{\mathrm{P}}\right.$ is the Planck time), $m=m_{e}$ ( $m_{e}$ is the mass of electron) the inequality can be rewritten as $\alpha^{2} m_{e} / 9 F_{\mathrm{P}}^{2} m_{\mathrm{P}}<1$, here $F_{\mathrm{P}}$ is the Planck force, $m_{\mathrm{P}}$ is the Planck mass. Note that this inequality is violated for very large $\alpha$, $\alpha \geq 3 F_{\mathrm{P}} \sqrt{m_{\mathrm{P}}} / \sqrt{m_{e}}=5,6 \cdot 10^{55} \mathrm{H}$. In this case, the one particle theory does not work. Therefore, it has to be studied separately.

The operator of coordinate $x_{3}$ and the operator of momentum $p_{3}$ in $\tilde{H}_{3}$ satisfy the ordinary commutation relations (11), (12). So, $\tilde{H}_{3}$ corresponds to the Hamiltonian of a particle in a uniform field in the ordinary space (in a space where the operators of coordinates and operators of momenta satisfy the ordinary commutation relations).

Introducing

$$
\begin{aligned}
& \tilde{p}_{1}^{a}=p_{1}^{a}-\frac{\alpha c_{\theta}}{2 \omega_{\mathrm{osc}} l_{\mathrm{P}}^{2}} p_{2}, \\
& \tilde{p}_{2}^{a}=p_{2}^{a}+\frac{\alpha c_{\theta}}{2 \omega_{\mathrm{osc}} l_{\mathrm{P}}^{2}} p_{1}, \\
& \tilde{p}_{3}^{a}=p_{3}^{a}
\end{aligned}
$$


one can write (20) as

$$
\tilde{H}_{\mathrm{osc}}=\frac{\left(\tilde{p}^{a}\right)^{2}}{2 m_{\mathrm{osc}}}+\frac{m_{\mathrm{osc}} \omega_{\mathrm{osc}}^{2} a^{2}}{2} .
$$

Operators $a_{i}$ and $\tilde{p}_{i}^{a}$ satisfy the ordinary commutation relations

$$
\begin{aligned}
& {\left[a_{i}, a_{j}\right]=\left[\tilde{p}_{i}^{a}, \tilde{p}_{j}^{a}\right]=0,} \\
& {\left[a_{i}, \tilde{p}_{j}^{a}\right]=i \hbar \delta_{i j},}
\end{aligned}
$$

Therefore operator (32) corresponds to the Hamiltonian of a three-dimensional harmonic oscillator with mass $m_{\text {osc }}$ and frequency $\omega_{\text {osc }}$ in the ordinary space. The spectrum of the oscillator in the ordinary space is well known. Let us recall that the frequency $\omega_{\text {osc }}$ is very large [22], and the oscillator put in the ground state remains in it. So, the oscillator energy is $3 \hbar \omega_{\text {osc }} / 2$.

Operators $\tilde{H}_{1}, \tilde{H}_{2}, \tilde{H}_{3}, \tilde{H}_{\text {osc }}$ commute with each other (see $(21),(27)$ ). So, the spectrum of a particle in a uniform field in a rotationally invariant and time reversal invariant noncommutative space reads

$$
\begin{aligned}
E & =\frac{\hbar^{2} k_{1}^{2}}{2 m}\left(1-\frac{\alpha^{2} c_{\theta}^{2} m}{4 \hbar \omega_{\mathrm{osc}} l_{\mathrm{P}}^{2}}\right)+\frac{\hbar^{2} k_{2}^{2}}{2 m}\left(1-\frac{\alpha^{2} c_{\theta}^{2} m}{4 \hbar \omega_{\mathrm{osc}} l_{\mathrm{P}}^{2}}\right) \\
& +E_{3}+\frac{3}{2} \hbar \omega_{\mathrm{osc}} .
\end{aligned}
$$

Note that the motion of a particle in the directions perpendicular to the field direction is free. In (35), $k_{1}$, $k_{2}$ denote the components of the wave vector which correspond to this motion, $E_{3}$ denotes continious eigenvalues of $\tilde{H}_{3}$. The last term in (35) corresponds to the ground state energy of the harmonic oscillator (32).

Let us also write the eigenfunctions of the total Hamiltonian (18). Because relations (21), (27) are satisfied, we can write

$$
\psi(\mathbf{x}, \mathbf{a})=\tilde{\psi}_{1}\left(x_{1}\right) \tilde{\psi}_{2}\left(x_{2}\right) \tilde{\psi}_{3}\left(x_{3}\right) \tilde{\psi}(\mathbf{a}),
$$

where $\tilde{\psi}_{i}\left(x_{i}\right)$ are eigenfunctions of $\tilde{H}_{i}$ given by (24)-(26). Note that $\psi^{(3)}\left(x_{3}\right)$ is the eigenfunction of a particle in a uniform field in the ordinary space, which is well known (see, for instance, [27]). It reads

$$
\psi^{(3)}\left(x_{3}\right)=\left(\frac{4 m^{2}}{\pi^{3} \alpha \hbar^{4}}\right)^{\frac{1}{6}} \Phi\left(\left(\frac{2 m \alpha}{\hbar^{2}}\right)^{\frac{1}{3}}\left(-x_{3}-\frac{E_{3}}{\alpha}\right)\right)
$$

here $\Phi$ is the Airy function

$$
\Phi(x)=\frac{1}{\sqrt{\pi}} \int_{0}^{\infty} \cos \left(\frac{t^{3}}{3}+t x\right) d t .
$$

Functions $\tilde{\psi}(\mathbf{a})$ in (36) are eigenfunctions of

$$
\begin{aligned}
H_{\mathrm{osc}}^{\prime} & =\frac{1}{2 m_{\mathrm{osc}}}\left(p_{1}^{a}-\frac{\alpha c_{\theta} \hbar k_{2}}{2 \omega_{\mathrm{osc}} l_{\mathrm{P}}^{2}}\right)^{2} \\
& +\frac{1}{2 m_{\mathrm{osc}}}\left(p_{2}^{a}+\frac{\alpha c_{\theta} \hbar k_{1}}{2 \omega_{\mathrm{osc}} l_{\mathrm{P}}^{2}}\right)^{2}+\frac{\left(p_{3}^{a}\right)^{2}}{2 m_{\mathrm{osc}}} \\
& +\frac{m_{\mathrm{osc}} \omega_{\mathrm{osc}}^{2} a_{1}^{2}}{2}+\frac{m_{\mathrm{osc}} \omega_{\mathrm{osc}}^{2} a_{2}^{2}}{2}+\frac{m_{\mathrm{osc}} \omega_{\mathrm{osc}}^{2} a_{3}^{2}}{2}
\end{aligned}
$$

Hamiltonian (39) was obtained by replacing $p_{1}$ and $p_{2}$ with $\hbar k_{1}, \hbar k_{2}$, respectively, in (20). The eigenfunction of (39) corresponding to the ground state reads

$$
\tilde{\psi}(\mathbf{a})=\frac{1}{\pi^{\frac{3}{4}} l_{\mathrm{P}}^{\frac{3}{2}}} e^{-\frac{a^{2}}{2 l_{\mathrm{P}}^{2}}-i \beta\left(k_{1} a_{2}-k_{2} a_{1}\right)} .
$$

Here for convenience we use the following notation

$$
\beta=\frac{\alpha c_{\theta}}{2 \omega_{\mathrm{osc}} l_{\mathrm{P}}^{2}} \text {. }
$$

So, we can write the eigenfunctions of the total Hamiltonian (18). They read

$$
\begin{aligned}
& \psi(\mathbf{x}, \mathbf{a})=C e^{i k_{1} x_{1}} e^{i k_{2} x_{2}} \\
& \times \Phi\left(\left(\frac{2 m \alpha}{\hbar^{2}}\right)^{\frac{1}{3}}\left(-x_{3}-\frac{E_{3}}{\alpha}\right)\right) e^{-\frac{a^{2}}{2 l_{\mathrm{P}}^{2}}-i \beta\left(k_{1} a_{2}-k_{2} a_{1}\right)},
\end{aligned}
$$

where $C$ is the normalization constant.

Let us analyze the obtained results. It is important to note that the features of the space structure on the Planck scale have an effect only on the motion of a particle in the directions perpendicular to the direction of the field. The first two terms in (35) can be rewritten using effective mass (28). So, the space quantization has an effect on the mass of the particle in a uniform field in a rotationally invariant and time reversal invariant space with the noncommutativity of coordinates.

\section{CONCLUSION}

In the paper, we have considered an algebra with the noncommutativity of coordinates which is rotationally and time reversal invariant (8), (9). This algebra describes space quantization at the Planck scale. The influence of space quantization on the motion of a particle in a uniform field has been studied. Taking into account that the rotationally invariant and time reversal invariant noncommutative algebra contains additional momenta, we have constructed and examined a total Hamiltonian of a particle in a uniform field in a time reversal invariant and rotationally invariant noncommutative space (16). The energy and wave functions of the particle have been found exactly $(35),(43)$. We obtain that the motion of a particle in the field direction in a rotationally and time-reversal invariant noncommutative space is the same as in a space with ordinary commutation relations for operators of coordinates and operators of momenta. Features of the space structure described by noncommutative algebra (8), (9) have an effect on the motion of a particle in the directions perpendicular to the field direction. Similarly as in the ordinary space, the motion of a particle in these directions is free. The noncommutativity has only an effect on the particle mass.

Acknowledgement. The authors thank Prof. Tkachuk V. M. for his advice and support during the research studies. This work was partly supported by the Project $\Phi \Phi-11 \mathrm{Hp}$ (No. 0121U100058) from the Ministry of Education and Science of Ukraine. 
[1] N. Seiberg, E. Witten, J. High Energy Phys. 1999, 032 (1999); https://doi.org/10.1088/1126-6708/19 99/09/032.

[2] S. Doplicher, K. Fredenhagen, J. E. Roberts, Phys. Lett. B 331, 39 (1994); https://doi.org/10.1016/0370-269 3(94) 90940- 7 .

[3] J. Gamboa, M. Loewe, J. C. Rojas, Phys. Rev. D 64, 067901 (2001); https://doi.org/10.1103/PhysRevD.6 4.067901.

[4] V. P. Nair, A. P. Polychronakos, Phys. Lett. B 505, 267 (2001); https://doi.org/10.1016/S0370-2693(01) 003 39- 2 .

[5] M. Chaichian, M. M. Sheikh-Jabbari, A. Tureanu, Phys. Rev. Lett. 86, 2716 (2001); https ://doi .org/10.1103/ PhysRevLett.86.2716.

[6] S. Biswas, P. Nandi, B. Chakraborty, Phys. Rev. A 102, 022231 (2020); https://doi.org/10.1103/PhysRevA.1 02.022231 .

[7] J. Lukierski, M. Woronowicz Phys. Lett. B 633, 116 (2006); https://doi.org/10.1016/j.physletb.2 005.11 .052 .

[8] G. Amelino-Camelia, M. Arzano, Phys. Rev. D 65, 084044 (2002); https://doi.org/10.1103/PhysRevD.6 5.084044 .

[9] M. Daszkiewicz, C. J. Walczyk, Phys. Rev. D 77, 105008 (2008); https://doi.org/10.1103/PhysRevD.7 7.105008 .

[10] L. N. Chang, D. Minic, N. Okamura, T. Takeuchi, Phys. Rev. D 65, 125027 (2002); https://doi.org/10.1103/ PhysRevD.65.125027.

[11] S. Benczik, L. N. Chang, D. Minic, T. Takeuchi, Phys. Rev. A 72, 012104 (2005); https://doi.org/10.1103/ PhysRevA.72.012104.

[12] C. Quesne, V. M. Tkachuk, Phys. Rev. A 81, 012106 (2010); https://doi.org/10.1103/PhysRevA.8 1.012106.

[13] M. Schneider, A. DeBenedictis, Phys. Rev. D 102, 024030 (2020); https://doi.org/10.1103/PhysRevD.1 02.024030 .

[14] O. Bertolami, J. G. Rosa, C. M. L. de Aragão, P. Castori- na, D. Zappalà Phys. Rev. D 72, 025010 (2005); https: //doi.org/10.1103/PhysRevD.72.025010.

[15] O. Bertolami, R. Queiroz, Phys. Lett. A 375, 4116 (2011); https://doi.org/10.1016/j.physleta.2011. 09.053.

[16] J. M. Romero, J. D. Vergara Mod. Phys. Lett. A 18, 1673 (2003); https://doi.org/10.1142/S02177323030 11472.

[17] B. Mirza, M. Dehghani, Commun. Theor. Phys. 42, 183 (2004); https://doi.org/10.1088/0253-6102/42 /2/183.

[18] C. Bastos, O. Bertolami, Phys. Lett. A 372, 5556 (2008); https://doi.org/10.1016/j.physleta. 2008.06.073.

[19] K. H. C. Castello-Branco, A. G. Martins, J. Math. Phys. 51, 102106 (2010); https://doi.org/10.1063/1.3466 812.

[20] J. Ben Geloun, S. Gangopadhyay, F. G. Scholtz, Europhys. Lett. 86, 51001 (2009); https://doi.org/10 .1209/0295-5075/86/51001.

[21] F. G. Scholtz, L. Gouba, A. Hafver, C. M. Rohwer, J. Phys. A 42, 175303 (2009); https://doi.org/10.1088/ 1751-8113/42/17/175303.

[22] Kh. P. Gnatenko, M. I. Samar, V. M. Tkachuk, Phys. Rev. A 99, 012114 (2019); https://doi.org/10.1103/ PhysRevA.99.012114.

[23] Kh. P. Gnatenko, V. M. Tkachuk, Phys. Lett. A 378 , 3509 (2014); https://doi.org/10.1016/j.physleta.2 014.10 .021$.

[24] Kh. P. Gnatenko, V. M. Tkachuk, Mod. Phys. Lett. A 31, 1650026 (2016); https://doi.org/10.1142/S02177 32316500267.

[25] Kh. P. Gnatenko, V. M. Tkachuk, Int. J. Mod. Phys. A 33, 1850037 (2018); https://doi.org/10.1142/S02177 $51 \times 18500379$.

[26] Kh. P. Gnatenko, O. V. Shyiko, Mod. Phys. Lett. A 33, 1850091 (2018); https ://doi .org/10.1142/S021773231 8500918.

[27] L. D. Landau, E. M. Lifshitz, Quantum Mechanics NonRelativistic Theory, Course of Theoretical Physics. Vol. 3 (Pergamon Press, Oxford, 1977).

\title{
ЧАСТИНКА В ОДНОРІДНОМУ ПОЛІ В НЕКОМУТАТИВНОМУ ПРОСТОРІ ЗІ ЗБЕРЕЖЕНОЮ СИМЕТРІЕЮ ВІДНОСНО ІНВЕРСІЇ ЧАСУ ТА СФЕРИЧНОЮ СИМЕТРІЕЮ
}

\author{
Х. П. Гнатенко, Х. І. Стахур, А. В. Крижова \\ Лъвівсъкий начіоналъний університет імені Івана Франка, кафедра теоретичной фізики, \\ вул. Драгоманова, 12, 79005 Лъвів, Украӥна
}

Вивчено вплив квантованості простору на рух частинки в однорідному полі. Для опису особливостей структури простору на планківських масштабах та врахування квантованості простору розглянуто ідею деформації звичних комутаційних співвідношень для операторів координат. А саме: припускається, що комутатор координат не дорівнює нулеві. У літературі відомо багато різних типів деформацій звичних комутаційних співвідношень для операторів координат та імпульсів. Найпростішою та найбільш вивченою $є$ алгебра з некомутативністю координат канонічного типу. Вона описує квантований простір, але зумовлює порушення симетрії відносно інверсії часу, порушення сферичної симетрії. У статті досліджено інваріантну відносно інверсії часу та сферично-симетричну алгебру 3 некомутативністю координат канонічного типу, яка описує простір із мінімальною довжиною (квантований простір) та була запропонована в роботі [Kh. P. Gnatenko, M. I. Samar, V. M. Tkachuk, Phys. Rev. A 99, 012114 (2019)]. Алгебра характеризується тензором некомутативності, побудованим за допомогою додаткових імпульсів. Останні відповідають сферично-симетричній системі. Розглянуто випадок, коли ця система - це гармонічний осцилятор із довжиною, що дорівнює довжині Планка, 
та великою частотою. Досліджено частинку в однорідному полі у квантованому просторі зі збереженою симетрією відносно інверсії часу та сферичною симетрією. Ми побудували та проаналізували повний гамільтоніан частинки в однорідному полі. Використавши метод зображення некомутативних координат через координати та імпульси, які задовольняють звичні комутаційні співвідношення, ми знайшли точний вираз для енергії та хвильових функцій частинки в однорідному полі. У статті показано, що рух частинки в напрямку однорідного поля у сферично-симетричному та інваріантному відносно інверсії часу просторі з некомутативністю координат є таким самим, як у звичному просторі. Некомутативність координат впливає тільки на рух частинки в напрямках, перпендикулярних до поля. А саме: квантованість простору впливає на масу частинки.

Ключові слова: квантований простір, некомутативні координати, симетрія відносно інверсії часу, сферична симетрія, частинка в однорідному полі. 